\title{
Streptococcal IdeS and Its Impact on Immune Response and Inflammation
}

\author{
Ulrich von Pawel-Rammingen \\ Department of Molecular Biology and Umeå Centre for Microbial Research, Umeå University, Umeå, Sweden
}

\section{Key Words}

Cysteine protease $\cdot$ Streptococcus pyogenes .

Immunoglobulin $\mathrm{G} \cdot$ Neutrophil priming

\begin{abstract}
Survival of the important bacterial pathogen Streptococcus pyogenes relies on its ability to circumvent the antimicrobial actions of innate and specific immune responses and to modulate the inflammatory responses induced during the course of an infection. Inflammatory processes play key roles during streptococcal pathogenesis and streptococcal infections are accompanied by an intense inflammatory state. As an exclusively human pathogen, S. pyogenes has adapted to the various countermeasures employed by its host to fight bacterial infections, in particular to interfere with the effector functions of immunoglobulin $\mathrm{G}(\mathrm{lgG})$. For this purpose, $S$. pyogenes has evolved an IgG-specific endopeptidase, IdeS, which is highly specific for the lower hinge region of $\operatorname{lgG}$. This review summarizes the current knowledge about this intriguing enzyme as well as its role in inflammation and in the attenuation of human immune responses towards streptococcal infection.

Copyright $\odot 2012$ S. Karger AG, Basel
\end{abstract}

\section{Introduction}

Pathogenic bacteria have evolved various strategies to invade their host, to acquire nutrients from diverse food sources and to establish themselves in one or several microenvironments within the host. A distinct set of virulence factors is employed to promote growth and to allow the pathogens to compete with commensal bacteria, but, most importantly, to enable the bacteria to escape from host immune responses. The Gram-positive bacterium Streptococcus pyogenes is one of the most common and important human bacterial pathogens. Streptococcal infections include throat and skin infections, as well as invasive, life-threatening conditions such as necrotizing fasciitis and toxic shock syndrome [1]. Mucocutaneous infections cause substantial morbidity and considerable economic loss to society, as more than 600 million people are estimated to suffer from streptococcal pharyngitis alone [2]. Streptococcal infections also harbor a risk for postinfectious sequelae, including serious inflammatory/autoimmune diseases such as acute rheumatic fever, rheumatic heart disease and post-streptococcal glomerulonephritis. In addition, other inflammatory autoimmune diseases, e.g. guttate

Dr. Ulrich von Pawel-Rammingen

Department of Molecular Biology

Umeå University

SE-90187 Umeå (Sweden)

Tel. +4690785 0808, E-Mail ulrich.von-pawel@molbiol.umu.se 
psoriasis, have also been associated with streptococcal infections [3], although the underlying molecular mechanisms are still elusive.

S. pyogenes infections are commonly associated with an intense inflammatory state [4], reflecting an increased vascular permeability and recruitment of neutrophils to the site of infection. However, to view inflammatory responses only as a host-protective measure to fight bacterial infection does not give a justified picture of the complex host-parasite relationship. Inflammatory responses, the activation of complement cascades and the degranulation of neutrophils have, of course, protective functions, but also lead to tissue damage and, in serious disease, to the loss of function of both tissues and organs. Increased vascular permeability, stimulated exudate flow and tissue damage are also important parameters for the spread of bacteria and supplying of nutrients. Thus, the survival of $S$. pyogenes does rely on its ability to modulate these inflammatory responses to a level that is beneficial for the bacteria at the same time as the bacteria have to avoid the various actions of host immunity. Although asymptomatic carriage of $S$. pyogenes is common, as an exclusively human pathogen it is likely to eventually cause symptoms and to induce an immune response and protective immunity. Since protective immunity harbors the obvious risk of the extinction of the pathogen in host populations, $S$. pyogenes has not only to deal with innate immune responses but also with specific antibodies. IgA is the abundant antibody in mucosal surfaces and plays an important role by protecting mucosal surfaces against bacterial colonization and invasion, and also by interfering with the action of microbial virulence factors $[5,6]$. The effector functions of IgA depend on the structural integrity of antigen - and effector domains and many pathogenic bacteria including certain species of Streptococcus secrete IgA1 proteases [7]. The proteolytic activity of $\operatorname{IgA}$ proteases is not restricted to $\operatorname{Ig} \mathrm{A}$, as the enzymes have also been shown to cleave other proteins, e.g. LAMP1, a phagosomal membrane glycoprotein [8].

Although $S$. pyogenes frequently colonizes mucosal surfaces, the bacteria apparently lack IgA proteases but employ an IgA-binding $M$ protein to interfere with the binding of IgA to its receptors [9]. However, as mentioned above, upon infection and inflammation, a plasma-exudative response is mounted [10] and the pathogen has to deal with both specific immunoglobulin $G$ (IgG) and neutrophils that are recruited to the infection site.

S. pyogenes has evolved a highly specific IgG endopeptidase to accomplish this task. This enzyme, designated IdeS, has unique properties in that it not only degrades
IgG, but also directly and indirectly represses innate immune responses to promote streptococcal survival in an inflammatory environment.

\section{Streptococcal IgG-Degrading Enzyme of S. pyogenes}

Ig-degrading activity is a rather common strategy employed by pathogenic bacteria to avoid the detrimental activity of specific IgG [11]. In S. pyogenes, the classic cysteine protease SpeB [12] has been described as cleaving IgG and $\operatorname{IgA}$ in the flexible hinge region $[13,14]$ and thereby interfering with the effector functions of IgG. However, proteolytic cleavage of IgG by SpeB is very slow and appears not suitable to deal with specific antibodies [15]. A much more powerful IgG-cleaving activity was discovered when investigating proteolytic activities in streptococcal growth medium supplemented with human plasma [16]. Proteolytic activity against IgG was assigned to a secreted $35 \mathrm{kDa}$ protein and called IgG-degrading enzyme of S. pyogenes (IdeS) [16]. IdeS was found to be identical to streptococcal Mac-1, a protein that had been proposed to inhibit phagocytosis by inhibiting Fc receptor (CD16) recognition of IgG and/or complement disposition $[17,18]$. Subsequent studies revealed that IdeS/Mac-1 can indeed inhibit certain neutrophil effector functions, i.e. the production of reactive oxygen species (ROS), independently from IgG endopeptidase activity, but also that this inhibition per se is not sufficient to mediate streptococcal survival in bactericidal assays [19] (see below).

Two allelic variants of the ides gene have been described [20] and one or the other of the two variants is encoded in all S. pyogenes strains investigated $[16,18,21$, 22], including clinically important serotypes, e.g. M1, M3, M4 and M12 as well as more recently validated serotypes, like M122 [20, 21]. The fact that the protease is conservatively encoded in $S$. pyogenes indicates an important role of IdeS for streptococcal pathogenicity. Although the two variants differ more than $50 \%$ in amino acid sequences in the middle third of the protein (amino acids 112-205) [20], both forms encode enzymes with pronounced IgG-specific endopeptidase activity [19]. To distinguish the two allelic forms, the first variant is now commonly designated IdeS (sometimes Mac-1), while the second variant is called Mac-2. Enzymatic activity of Mac-2 proteins is indistinguishable from the enzymatic activity of IdeS proteins [19] and an earlier report of only weak IgG endopeptidase activity for Mac-2 [20] is only valid for proteins of the streptococcal M28 serotype [21]. Mac-2 proteins of this serotype represent an unique ex- 
ception in that the presence of a second cysteine residue mediates the formation of a disulfide bond to the catalytic site cysteine of Mac- $2^{\mathrm{M} 28}$, thereby interfering with substrate recognition and/or cleavage [19]. Thus, with the exception of Mac- $2^{\mathrm{M} 28}$, both IdeS and Mac-2 protein variants are bifunctional proteins that combine both $\mathrm{Fc}$ receptor-binding activity and IgG endopeptidase activity to achieve efficient protection against IgG-mediated phagocytosis [19].

\section{The IdeS Protease Family}

IdeS homologs have been identified in other streptococcal species: S. equi ssp equi and S. equi ssp zooepidemicus encode two alleles designated ide $\mathrm{E}$ and ide $\mathrm{E} 2$ and ideZ and ideZ2, respectively [23, 24]. The S. equi homologs IdeE and IdeZ share $99 \%$ amino acid sequence identity with each other and are more closely related to the Mac-2 protein of S. pyogenes than to IdeS [20]. IdeE2 and IdeZ2 share $70 \%$ sequence identity with each other, but only about $25 \%$ with IdeE, IdeZ, IdeS or Mac-2 [24]. All four alleles encode proteins with IgG endopeptidase activity, although to different degrees and specificities [24]. Interestingly, IdeE and IdeZ exhibit only weak proteolytic activity towards horse IgG, but are efficient in cleaving human IgG [23]. Furthermore, they are also weakly active against mouse IgG. They are equally efficient at cleaving horse and human IgG, but lack the ability to cleave IgG from mouse, rabbit and sheep [24]. An IdeS-like domain is also present in the approximately 110 $\mathrm{kDa}$ Mac family protein from S. suis [25], but putative activities and functions of this protein have so far not been characterized. An IdeS-like domain has been identified in the IdeT protein of Treponema denticola, a Gramnegative oral spirochete frequently associated with chronic periodontitis [26]. The IdeS-like domain has been designated dentipain and exhibits approximately 25\% identity to IdeS and IdeE but lacks Ig-degrading activity. However, dentipain has proteolytic activity as an oligopeptidase [26].

\section{Hydrolysis of IgG}

IgG hydrolysis occurs at one defined site between the two glycine residues in positions 236 and 237 in the lower hinge region of the IgG heavy chain. The lower hinge region of IgG has been implicated as being important for Fc receptor recognition and complement binding $[27,28]$.
Thus, proteolytic cleavage in this region will not only effectively prevent the IgG-mediated recruitment of immune cells to the bacteria, but is also likely to interfere with the recognition of IgG (fragments) by complement and/or Fc receptors (see below). As a consequence of cleavage in the lower hinge region, IdeS generates one $\mathrm{F}\left(\mathrm{ab}^{\prime}\right)_{2}$ fragment and two identical $1 / 2 \mathrm{Fc}$ fragments [16], which is in contrast to the activity of papain that cleaves in the upper hinge region of IgG, yielding a $50 \mathrm{kDa}$ $\mathrm{Fc}_{\text {papain }}$ fragment. The papain derived $50 \mathrm{kDa} \mathrm{Fc}_{\text {papain }}$ fragment is, to date, the smallest known substrate of IdeS [29]. Cleavage of IgG occurs in two steps, where cleavage of one heavy chain is significantly faster than cleavage of the second heavy chain $[30,31]$. Single-cleaved IgG is in a physiological environment indistinguishable from intact IgG and has the same half-life in vivo as uncleaved IgG [31]. However, most importantly, single-cleaved IgG has a significantly lower affinity for Fc $\gamma$ receptors and can no longer mediate the effector functions of IgG [31]. Enzyme kinetic analysis of IdeS activity towards IgG demonstrated a non-Michaelis-Menten-like velocity curve over a broad concentration range of the enzyme $[29,32]$. The sigmoidal-shaped curves might be explained by the presence of two cooperative binding sites [29]. Two binding sites acting together in substrate recognition can be accomplished either by two recognition motifs present in monomeric IdeS, i.e. the catalytic site and a second socalled exosite, located in different parts of the IdeS molecule, or by the assumption that the catalytic sites of two IdeS molecules act together in a dimeric form of IdeS [29, 33]. Dimeric molecules have, in fact, been reported for crystal forms of IdeS [30], but bioinformatic analyses and experimental evidence strongly suggest that the dimeric form of IdeS is rather due to crystal packaging than representing a biological active form [34] (Vindebro et al., unpubl. data). The crystal structure of IdeS [35] reveals the typical structural fold of the papain superfamily of cysteine proteases, in which the active site is embedded in a distinct cleft between two globular domains [35]. The active site encompasses a classic catalytic triad in which the catalytic Cys94 is hydrogen-bonded to His262 and completed by a hydrogen bond between Asp284 and His262. This conformation, together with Lys84 and Asp286, forms a negatively charged region that has been implicated as being important for the proper positioning of IgG in the active site [35]. These data are consistent with previous results from enzymatic assays that revealed His262, Asp284 and Asp 286 to be important for the enzymatic activity of the enzyme [36]. Interestingly, IdeS carries an Arg-Gly-Asp motif [20]. The motif is located 
on a surface loop in the N-terminal region of IdeS, indicating that this classic integrin-binding motif does not interfere with proteolytic activity [33]. Instead, the ArgGly-Asp motif of IdeS and Mac-2 mediates interaction with $\alpha \mathrm{V} \beta I I I$ and $\alpha \mathrm{IIb} \beta 3$ integrins in experimental assays, although the putative role of this interaction is still unknown [20].

\section{Powering IdeS Activity}

Although secreted IdeS is a highly active and efficient IgG endopeptidase, considering the vast excess of IgG in the circulation, protection against specific IgG appears to be an overwhelming task. While it can easily be imagined that IdeS concentrations are sufficiently high to mediate fast proteolysis of specific IgG in the vicinity of the infection focus, exudate influx and radial diffusion of the enzyme will increasingly dilute enzyme concentrations. If enzyme concentrations become too diluted, IdeS activity is not sufficient to maintain effective protection. Under such conditions, endogenous protease inhibitors, like the ubiquitous human protease inhibitor cystatin C [37], are expected to counteract IdeS activity (as previously described for other microbial proteases) [36-40]. This is, however, not the case: instead, human cystatin $\mathrm{C}$ was identified as a natural, facultative cofactor of IdeS. IdeS activity is not dependent on cystatin C, but the latter significantly accelerates the enzymatic velocity of the protease [32] (fig. 1A). As cystatin $\mathrm{C}$ is widely distributed in body liquids and tissues, $S$. pyogenes has the ability to maintain a high activity of IgG endopeptidase and protection against specific IgG even at low enzyme concentrations, e.g. at a distance from the infection focus or early on in infection. Acceleration of a streptococcal cysteine protease by an abundant human cysteine protease inhibitor is a unique example of the coevolutionary processes between $S$. pyogenes and the human host [32]. The originally proposed model for the stimulatory activity of cystatin $\mathrm{C}$ was a mechanism where the inhibitor would promote IdeS dimerization [32], which in turn was suggested to be increasing IgG endopeptidase activity [33]. In the light of recent experimental results, however, it is not likely that IdeS forms a dimer (Vindebro et al., unpubl. data) or that a putative dimer would be enzymatically more active than monomeric IdeS. Acceleration of enzyme activity is not due to increased substrate recognition or product dissociation [33], and alternative explanations for the stimulatory activity of cystatin $\mathrm{C}$ on IdeS are currently under investigation.

Streptococcal IdeS

\section{IdeS Activity and Nonimmune Binding of IgG}

S. pyogenes has evolved M protein and M-like proteins that are major virulence factors and act as bacterial Fc receptors at the streptococcal cell surface. Nonimmune Fc binding mediates the immune evasion of specific IgG by counteracting signaling to immune cells and complement factors, i.e. by blockage of Fc $\gamma$ receptor binding [41, 42] and by inhibition of complement activation through the classic pathway [43]. However, in light of the vast amounts of IgG present in the circulation, the capacity of $S$. pyogenes to bind $\mathrm{IgG}$ is limited and the majority of Fc binding sites at the streptococcal surface are occupied by nonspecific IgG, leaving the bacteria vulnerable to the detrimental action of specific antibodies. In addition, binding of IgG to the streptococcal M proteins is in equilibrium and thus, per se, not designed to mediate longterm protection against specific antibodies [44]. Such antibodies will eventually dissociate and hence be able to opsonize the bacterium and mediate its killing. A longlasting and more efficient evasion of specific IgG becomes possible through the secretion of IdeS (fig. 1B, a). The protease potentiates the effect of nonimmune binding of IgG in various ways: (1) in an environment with low amounts of IgG, the proteolytic activity of IdeS will be sufficient to diminish the overall load of specific IgG in the vicinity of the bacteria and hence increase the probability of quantitative binding of specific IgG in a nonimmune fashion (fig. 1B, b) and (2) nonimmune binding of IgG by $\mathrm{M}$ proteins is followed by the proteolytic cleavage of the bound antibody. IdeS-generated $1 / 2 \mathrm{Fc}$ fragments have a low affinity for $\mathrm{M}$ protein and do not compete efficiently with intact IgG in binding to the bacterial surface. Thus, rapid dissociation of $1 / 2 \mathrm{Fc}$ allows the binding (and inactivation) of new IgG (fig. 1B, c). The cooperative binding and cleavage of IgG increases the probability that the bacteria resist specific IgG, despite the presence of a large excess of nonspecific IgG in the circulation.

\section{Effects of IgG Degradation Products}

The extensive cleavage of IgG by IdeS results in the formation of high amounts of circulating $1 / 2 \mathrm{Fc}$ and $\mathrm{F}\left(\mathrm{ab}^{\prime}\right)_{2}$ fragments. $\mathrm{F}\left(\mathrm{ab}^{\prime}\right)_{2}$ fragments derived from specific antistreptococcal antibodies retain the ability to bind to surface antigens, but lack the ability to signal to immune cells or to activate the complement system. Thus, when bound to the streptococcal surface, these fragments interfere with the recognition of streptococcal surface an- 


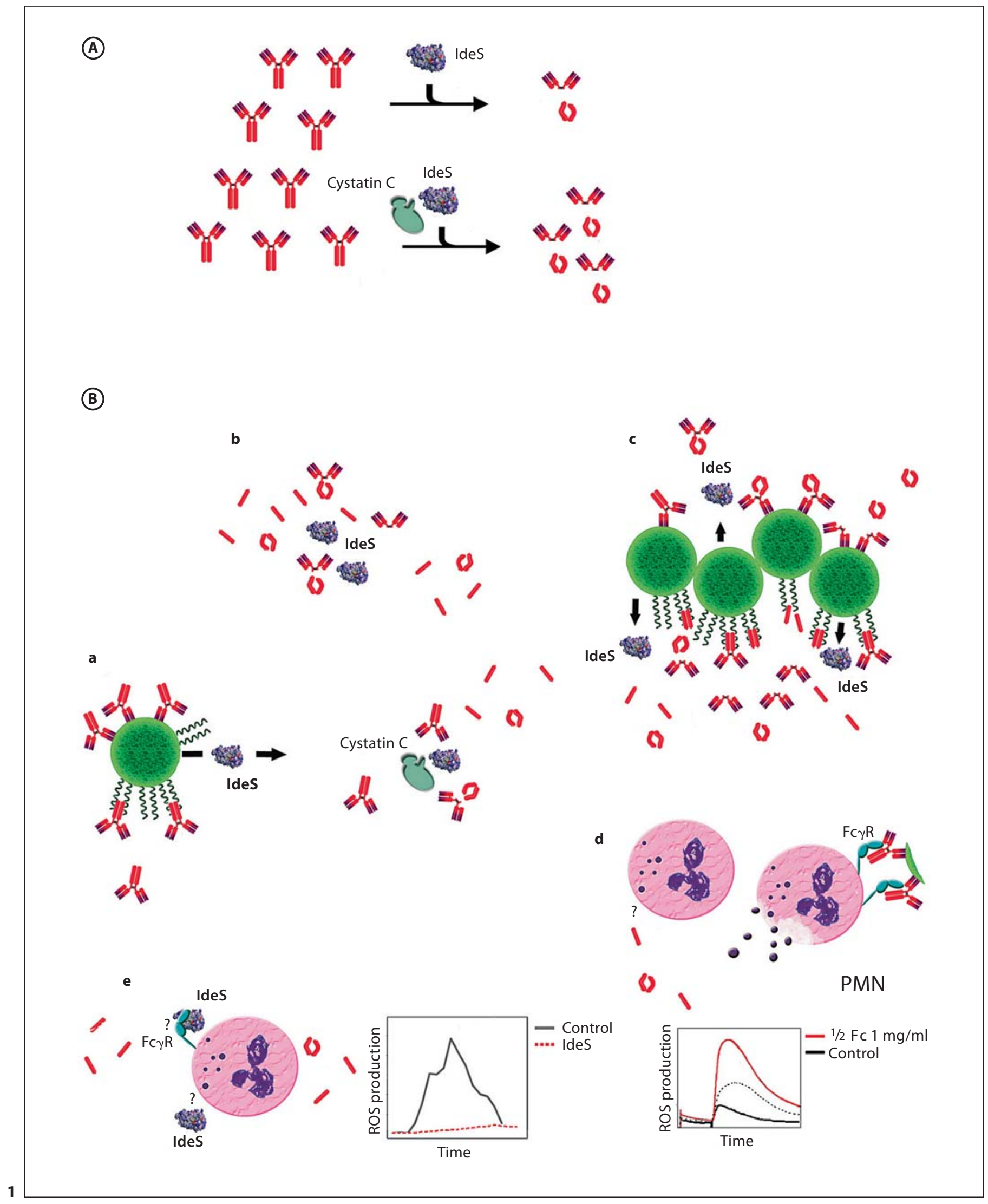


tigens by specific, uncleaved antibodies, exerting a protective effect. However, cleavage of unspecific IgG also generates $\mathrm{F}\left(\mathrm{ab}^{\prime}\right)_{2}$ fragments that are released in the circulation, but whether these fragments have a further biological effect has still to be determined. More information is available on the action of released $1 / 2 \mathrm{Fc}$ fragments; in an experimental setting in nonimmune blood, streptococcal survival decreased significantly when blood was preincubated with them [44]. This effect is accounted for by a mechanism known as 'priming', which is defined as PMN activation by a second stimulus with an enhanced rate and extent of ROS production by NADPH oxidase [45-47]. Upon activation of PMNs with opsonized latex beads, not only an increased maximum, but also a much earlier peak of ROS production was obtained in $1 / 2 \mathrm{Fc}$ primed samples (fig. $1 \mathrm{~B}, \mathrm{~d}$ ). The response from $1 / 2 \mathrm{Fc}$ primed PMNs is more powerful and considerably faster when compared to nonprimed cells. Thus, while the priming of PMNs by $1 / 2 \mathrm{Fc}$ fragments leads to the increased killing of $S$. pyogenes in experimental bactericidal assays, in the human host $1 / 2$ Fc-priming represents an important mechanism to promote bacterial survival during infection. In vivo, these fragments can freely diffuse from the site of bacterial colonization and thus prime PMNs at a distance from the site of infection. Once activated e.g. by immunocomplexes, these PMNs will discharge at a dis-

Fig. 1. Overview of features and functions of streptococcal IdeS. A Powering IdeS activity. Interaction of IdeS with the abundant cysteine protease inhibitor cystatin $\mathrm{C}$ accelerates IgG endopeptidase activity. B IdeS biological activity. a S. pyogenes, recognized by specific IgG and/or binding IgG to M protein at the streptococcal surface, secrete active IdeS. b In the circulation, IdeS cleaves $\mathrm{IgG}$ in the hinge region, generating single-cleaved inactive $\mathrm{IgG}$, $\mathrm{F}\left(\mathrm{ab}^{\prime}\right)_{2}$ fragments and two identical $1 / 2 \mathrm{Fc}$ fragments. c Specific IgG at the streptococcal surface is efficiently cleaved by IdeS, leaving antigen bound $\mathrm{F}\left(\mathrm{ab}^{\prime}\right)_{2}$ fragments that protect streptococcal surface antigens and releasing $1 / 2 \mathrm{Fc}$ fragments. Fc-bound $\mathrm{IgG}$ is also cleaved, releasing $\mathrm{F}\left(\mathrm{ab}^{\prime}\right)_{2}$ fragments and, due to their low affinity for $\mathrm{M}$-protein, $1 / 2 \mathrm{Fc}$ fragments into the circulation. Free binding sites at M protein can bind new IgG. d Circulating Fc fragments prime polymorphonuclear leukocytes (PMN) to an increased and fast production of ROS by binding to a yet-unknown receptor. Upon activation by immunocomplexes, these PMNs discharge and degranulate at a distance from the infection site without harming the bacteria. e Suppression of ROS production by IdeS to prevent PMNs to discharge close to the bacteria; IdeS suppresses ROS production, independently from its IgG endopeptidase activity. The precise mechanism so far remains elusive, but might be mediated by interaction with FcyR. tance from the bacteria without being harmful to the pathogen (fig. 1B, e). Activated PMNs will thereby contribute to inflammatory processes that facilitate the spread of $S$. pyogenes to new infection sites. Interestingly, to prevent PMNs from discharging close to the bacteria, IdeS itself interacts with PMNs, leading to the efficient suppression of ROS production [19]. The precise mechanism so far remains elusive, but it is not dependent on the IgG endopeptidase activity of IdeS [19], and might be mediated by interaction with FcyR [18]. Thus, close to the focus of infection, where IdeS concentrations are high, IdeS itself prevents ROS production in neutrophils and counteracts the priming effect of IgG $1 / 2 \mathrm{Fc}$ fragments.

\section{Host Immune Response to IdeS}

Analysis of the acute-phase serum samples of patients suffering from streptococcal disease demonstrated the presence of detectable antibody levels against IdeS $[17,18$, $22,48]$. These results correlate well with the fact that antibodies specific for IdeS are also widely distributed in the healthy population [22] and reflect a pre-existing immune response caused by previous $S$. pyogenes infections. In the majority of these patient samples, neutralizing activities could be detected in acute-phase serum, an important finding, as the level of neutralizing antibodies i.e. the antibody quality, has been suggested to be clinically more relevant than the quantity $[49,50]$. Furthermore, when investigating paired acute- and convalescent-phase serum samples from patients suffering from streptococcal tonsillitis or erysipelas, it was found that all patients that seroconvert to IdeS, i.e. those who developed antibodies towards IdeS, also obtained IgG antibodies with the ability to neutralize IdeS enzymatic activity [21]. Although no direct correlation between the presence or absence of neutralizing antibodies and the severity of invasive disease/disease outcome could be observed, neutralizing antibodies interfered with IdeS-mediated streptococcal survival ex vivo and significantly affected the ability of IdeS to interfere with Fc-mediated host defense mechanisms [21]. The importance of specific antibodies is underlined in two papers that used recombinant IdeS or the S. equi homologs IdeE and IdeE2 in immunization studies to successfully induce protective immunity against streptococci in mice $[24,48]$. As mouse IgG is only poorly, if at all, cleaved by these enzymes [24,51], the protective effect of immunization appears to act in a different manner on IdeS. One hypothesis might be that IdeS is surface-associated or retained at the streptococcal sur- 
face, so that specific antibodies opsonize the pathogen and mediate bacterial killing [24]. In fact, IdeS has been shown to be associated with the streptococcal surface in physiological environments (von Pawel-Rammingen, unpub. data). Another hypothesis might be that specific antibodies interfere with functions other than endopeptidase activity, e.g. the inhibition of ROS production [18, 44], or novel, as yet undiscovered activities of the protein. For instance, IdeS (designated Sib35 [52]) has been implicated as exerting a stimulatory mitogenic effect on B lymphocytes in an experimental mouse model [53].

\section{Counteracting IdeS Activity}

Endogenous protease inhibitors exert a tight control on proteolytic activities that might potentially become harmful for the living organism [37]. Several studies have shown that IdeS is active in physiological settings, e.g. in saliva, serum and plasma samples [21, 32, 54], and it appears that endogenous inhibitors are not effective or at least not present in concentrations high enough to interfere with IdeS activity in these environments. On the contrary, as mentioned above, protease activity was found to be markedly accelerated in the presence of human cystatin $\mathrm{C}$ and no endogenous, nonimmune inhibitor has yet been identified. Considering that IdeS has a powerful ability to interfere with IgG-mediated immune responses, there is potential medical interest to identify effective and specific inhibitors for this cysteine protease. However, to accomplish specific inhibition is not a simple task, due to the conserved structural similarity of papain-like proteases that include essential human cysteine proteases e.g. members of the cathepsin family. Despite these structural similarities to other papain-like protease, IdeS exhibits some distinct features that might allow the design of unique and specific inhibitors. Firstly, in contrast to most other eukaryotic and prokaryotic cysteine proteases, IdeS is not inhibited by the classic cysteine protease inhibitor E64 [16, 32], and secondly, it does not catalyze the hydrolysis of synthetic or natural peptides containing amino acids in the $\mathrm{P}_{4}-\mathrm{P}_{1}$ positions preceding the IdeS cleavage site within the IgG hinge region [32]. These findings suggest a more complex substrate recognition mechanism [32] and might present a target for inhibitory compounds. So far, two studies addressed the identification of specific inhibitors to IdeS. One employed analogs to the well-known serine protease inhibitors TPCK (tosyl phenylalanyl chloromethyl ketone) and TLCK (tosyl lysyl chloromethyl ketone) to demonstrate the reversible inhi- bition of IdeS by replacing the active $\alpha$-chloro-ketone by aldehyde [55]. In another study, peptide analogs based on the $\mathrm{P}_{4}-\mathrm{P}^{\prime}{ }_{4}$ residues of IgG and carrying a piperidine moiety in one of the two glycine residues of the cleavage site were investigated for their inhibitory activity on IdeS [56], with several being found to have significant inhibitory capacity independently of $\mathrm{N}$-terminal or $\mathrm{C}$-terminal amino acid extensions [56]. Importantly, different inhibitory profiles towards the classic streptococcal cysteine protease SpeB or papain [56] identified compounds that are putative specific inhibitors of IdeS and thus only have weak effects on papain and SpeB. Such inhibitors might be a first step towards the generation of compounds that have the potential to act specifically against IdeS and not against other cysteine proteases and could therefore have therapeutic potential [56].

\section{Therapeutic Use of IdeS}

Despite its essential role in fighting invading microorganisms, IgG is also largely involved in various autoimmune diseases. In these cases, IgG autoantibodies, i.e. antibodies reacting against human antigens, interfere with important cellular functions, misdirecting an immune response towards the body's own cells. Furthermore, although performing according to its intended function, the action of IgG is not desirable during organ transplantation where antibodies are involved in acute transplant rejection by the recipient. Thus, the efficient removal of pathogenic IgG is an important clinical challenge. Due to the unique substrate specificity of IdeS and its fast and efficient enzymatic reaction, the streptococcal protease harbors the potential to function as a therapeutic drug for clinical conditions involving pathogenic IgG. Animal models have been used to demonstrate that IgG can efficiently be removed from the circulation in vivo [57], as would be required for the treatment of acute transplant rejections. Animal models have also been employed for experimental IgG-mediated arthritis [58], glomerulonephritis [59] and thrombocytopenic purpura [57], to provide proof of principle for the use of IdeS as a therapeutic agent for treatment of IgG-mediated autoimmune disease.

\section{Conclusions}

Almost 10 years since the discovery of a powerful IgG endopeptidase activity in $S$. pyogenes, research on IdeS continues to reveal intriguing findings, gaining knowl- 
edge on enzymatic mechanisms, molecular pathogenhost interactions and the proper (or improper) functioning of immune responses. The direct interaction of IdeS with immune cells and host proteins and the secondary effects of IdeS and IdeS activity affect the functioning of several immune cells and provide and modulate inflammatory responses. Many molecular details have still to be characterized and expected discoveries will shed a new light on many apparently established mechanisms. In particular, the finding that dedicated human protease inhibitors stimulate pathogenic protease activity is captivating, not least because the precise mechanism, although yet not unraveled, might change the view on proteaseprotease inhibitor interactions also in other proteolytic systems.

\section{Acknowledgements}

This work was supported by the Swedish Research Council (project numbers 2006-4522 and 2009-4997) and Insamlingsstiftelsen at Umea University.

Reine Vindebro is acknowledged for his contribution to the art work and to valuable discussions.

\section{References}

1 Cunningham MW: Pathogenesis of group A streptococcal infections. Clin Microbiol Rev 2000;13:470-511.

2 Carapetis JR, Steer AC, Mulholl EK, Weber M: The global burden of group A streptococcal diseases. Lancet Infect Dis 2005;5:685694.

-3 Telfer NR, Chalmers RJ, Whale K, Coleman G: The role of streptococcal infection in the initiation of guttate psoriasis. Arch Dermatol 1992;128:39-42.

4 Rasmussen M, Björck L: Proteolysis and its regulation at the surface of Streptococcus pyogenes. Mol Microbiol 2002;43:537-544.

5 Macpherson AJ, Uhr T: Induction of protective IgA by intestinal dendritic cells carrying commensal bacteria. Science 2004;303: $1662-1665$.

-6 Kaetzel CS, Robinson JK, Chintalacharuvu KR, Vaerman JP, Lamm ME: The polymeric immunoglobulin receptor (secretory component) mediates transport of immune complexes across epithelial cells: a local defense function for IgA. Proc Natl Acad Sci USA 1991;88:8796-8800.

7 Mistry D, Stockley RA: IgA1 protease. Int J Biochem Cell Biol 2006;38:1244-1248.

8 Hauck CR, Meyer TF: The lysosomal/phagosomal membrane protein h-LAMP-1 is a target of the IgA1 protease of Neisseria gonorrhoeae. FEBS Letters 1997;405:86-90.

-9 Pleass RJ, Areschoug T, Lindahl G, Woof JM: Streptococcal IgA-binding proteins bind in the $\mathrm{C} \alpha 2-\mathrm{C} \alpha 3$ interdomain region and inhibit binding of IgA to human CD89. J Biol Chem 2001;276:8197-8204.

10 Persson CG, Erjefält JS, Greiff L, Erjefält I, Korsgren M, Lindén M, Sundler F, Andersson M, Svensson C: Contribution of plasmaderived molecules to mucosal immune defence, disease and repair in the airways. Scand J Immunol 1998;47:302-313.
11 Brezski RJ, Jordan RE: Cleavage of IgGs by proteases associated with invasive diseases: an evasion tactic against host immunity? MAbs 2010;2:212-220.

12 Elliott SD: A proteolytic enzyme produced by group A streptococci with special reference to its effect on the type-specific $M$ antigen. J Exp Med 1945;81:573-592.

13 Collin M, Olsén A: EndoS, novel secreted enzyme from Streptococcus pyogenes with endoglycosidase activity on human IgG. EMBO J 2001;20:3046-3055.

14 Eriksson A, Norgren M: Cleavage of antigenbound immunoglobulin $\mathrm{G}$ by SpeB contributes to streptococcal persistence in opsonizing blood. Infect Immun 2003;71:211-217.

15 von Pawel-Rammingen U, Björck L: IdeS and SpeB: immunoglobulin-degrading cysteine proteases of Streptococcus pyogenes. Curr Opin Microbiol 2003;6:50-55.

16 von Pawel-Rammingen U, Johansson BP, Björck L: IdeS, a novel streptococcal cysteine proteinase with unique specificity for immunoglobulin G. EMBO J 2002;21:1607-1615.

17 Lei B, Mackie S, Lukomski S, Musser JM: Identification and immunogenicity of group A streptococcus culture supernatant proteins. Infect Immun 2000;68:6807-6818

18 Lei B, DeLeo FR, Hoe NP, Graham MR, Mackie SM, Cole RL, Liu M, Hill HR, Low DE, Federle MJ, Scott JR, Musser JM: Evasion of human innate and acquired immunity by a bacterial homolog of CD11b that inhibits opsonophagocytosis. Nat Med 2001; 7:1298-1305.

-19 Johansson Söderberg J, Engström P, von Pawel-Rammingen U: The intrinsic IgG endopeptidase activity of streptococcal Mac-2 proteins implies a unique role for the enzymatically impaired Mac- 2 protein of M28 serotype strains. Infect Immun 2008;76:21832188
20 Lei B, DeLeo FR, Reid SD, Voyich JM, Magoun L, Liu M, Braughton KR, Ricklefs S, Hoe NP, Cole RL, Leong JM, Musser JM: Opsonophagocytosis-inhibiting Mac protein of group A streptococcus: identification and characteristics of two genetic complexes. Infect Immun 2002;12:6880-6890.

-21 Åkesson P, Moritz L, Truedsson M, Christensson B, von Pawel-Rammingen U: IdeS, a highly specific IgG-cleaving enzyme from Streptococcus pyogenes, is inhibited by specific IgG antibodies generated during infection. Infect Immun 2006;74:497-503.

22 Åkesson P, Rasmussen M, Mascini E, von Pawel-Rammingen U, Janulczyk R, Collin M, Olsén A, Mattsson E, Olsson ML, Björck L, Christensson B: Low antibody titers to cell wall-attached proteins of Streptococcus pyogenes predispose for severe invasive disease. Infect Dis 2004;189:797-804.

23 Lannergård J, Guss B: IdeE, an IgG endopeptidase of Streptococcus equi ssp equi. FEMS Microbiol Lett 2006;262:230-235.

24 Hulting G, Flock M, Fryckberg L, Lannergård J, Flock JI, Guss B: Two novel IgG endopeptidases of Streptococcus equi. FEMS Microbiol Lett 2009;298:44-50.

-25 Holden MT, Hauser H, Sanders M, Ngo TH, Cherevach I, Cronin A, Goodhead I, Mungall K, Quail MA, Price C, Rabbinowitsch E, Sharp S, Croucher NJ, Chieu TB, Mai NT, Diep TS, Chinh NT, Kehoe M, Leigh JA, Ward PN, Dowson CG, Whatmore AM, Chanter N, Iversen P, Gottschalk M, Slater JD, Smith HE, Spratt BG, Xu J, Ye C, Bentley S, Barrell BG, Schultsz C, Maskell DJ, Parkhill J: Rapid evolution of virulence and drug resistance in the emerging zoonotic pathogen Streptococcus suis. PLoS One 2009; 4:e6072.

26 Ishihara K, Wawrzonek K, Shaw LN, Inagaki S, Miyamoto M, Potempa J: Dentipain, a Streptococcus pyogenes IdeS protease homo$\log$, is a novel virulence factor of Treponema denticola. Biol Chem 2010;391:1047-1055. 
27 Radaev S, Sun P: Recognition of immunoglobulins by Fc gamma receptors. Mol Immunol 2002;38:1073-1083.

28 Duncan AR, Winter G: The binding site for Clq on IgG. Nature 1988;332:738-740.

29 Vincents B, von Pawel-Rammingen U, Björck L, Abrahamson M: Biochemical characterization of IdeS, an IgG-specific endopeptidase from Streptococcus pyogenes. Biochemistry 2004;43:15540-15549.

- 30 Ryan MH, Petrone D, Nemeth JF, Barnathan E, Björck L, Jordan RE: Proteolysis of purified IgGs by human and bacterial enzymes in vitro and the detection of specific proteolytic fragments of endogenous IgG in rheumatoid synovial fluid. Mol Immunol 2008;45: 1837-1846.

- 31 Brezski RJ, Vafa O, Petrone D, Tam SH, Powers G, Ryan MH, Luongo JL, Oberholtzer A, Knight DM, Jordan RE: Tumor-associated and microbial proteases compromise host IgG effector functions by a single cleavage proximal to the hinge. Proc Natl Acad Sci USA 2009; 106:17864-17869.

- 32 Vincents B, Vindebro R, Abrahamsson M, von Pawel-Rammingen U: Acceleration of cysteine protease activity by the human protease inhibitor cystatin C. Chem Biol 2008; 15:960-968.

33 Agniswamy J, Nagiec MJ, Liu M, Schuck P, Musser JM, Sun PD: Crystal structure of group A streptococcus Mac-1: insight into dimer-mediated specificity for recognition of human IgG. Structure 2006;14:225-235.

- 34 Olsen JG, Dagil R, Niclasen LM, Sørensen OE, Kragelund BB: Structure of the mature streptococcal cysteine protease exotoxin $\mathrm{mSpeB}$ in its active dimeric form. J Mol Biol 2009;393:693-703.

- 35 Wenig K, Chatwell L, von Pawel-Rammingen U, Björck L, Huber R, Sondermann P: Structure of the streptococcal endopeptidase IdeS, a novel cysteine proteinase with strict specificity for IgG. Proc Natl Acad Sci USA 2004;101:17371-17376.

- 36 Lei B, Liu M, Meyers EG, Manning HM, Nagiec MJ, Musser JM: Histidine and aspartic acid residues important for immunoglobulin $\mathrm{G}$ endopeptidase activity of the group A streptococcus opsonophagocytosis-inhibiting Mac protein. Infect Immun 2003;5:28812884.

- 37 Rawlings ND, Tolle DP, Barrett AJ: MEROPS: a peptidase database. Nucleic Acids Res 2004; 32:D160-D164.
38 Turk B, Turk D, Salvesen GS: Regulating cysteine protease activity: essential role of protease inhibitors as guardians and regulators. Curr Pharm Des 2002;8:1623-1637.

39 Scharfstein J: Parasite cysteine protease interactions with alpha2-macroglobulin or kininogens: differential pathways modulating inflammation and innate immunity in infection by pathogenic trypanosomatids. Immunobiology 2006;211:117-125.

40 Björck L, Grubb A, Kjellén L: Cystatin C, a human protease inhibitor, blocks replication of Herpes simplex virus. J Virol 1990;64: 941-943.

41 Dossett JH, Kronvall G, Williams RC Jr, Quie PG: Antiphagocytic effects of staphylococcal protein A. J Immunol 1969;103:14051410.

42 Pleass RJ, Areschoug T, Lindahl G, Woof JM: Streptococcal IgA-binding proteins bind in the Calpha 2-Calpha 3 interdomain region and inhibit binding of IgA to human CD89. J Biol Chem 2001;276:8197-8204.

43 Berge A, Kihlberg BM, Sjöholm AG, Björck $\mathrm{L}$ : Streptococcal protein $\mathrm{H}$ forms soluble complement-activating complexes with IgG, but inhibits complement activation by IgGcoated targets. J Biol Chem 1997;272:2077420781.

44 Johansson Söderberg J, von Pawel-Rammingen U: The streptococcal protease IdeS modulates bacterial IgGFc binding and generates $1 / 2 \mathrm{Fc}$ fragments with the ability to prime polymorphonuclear leucocytes. Mol Immunol 2008;45:3347-3353.

45 McPhail LC, Clayton CC, Snyderman R: The NADPH oxidase of human polymorphonuclear leukocytes: evidence for regulation by multiple signals. J Biol Chem 1984;259:57685775.

46 Sheppard FR, Kelher MR, Moore EE, McLaughlin NJ, Banerjee A, Silliman CC: Structural organization of the neutrophil NADPH oxidase: phosphorylation and translocation during priming and activation. J Leukoc Biol 2005;78:1025-1042.

47 Swain SD, Rohn TT, Quinn MT: Neutrophil priming in host defense: role of oxidants as priming agents. Antioxid Redox Signal 2002; 4:69-83.

48 Okamoto S, Tamura Y, Terao Y, Hamada S, Kawabata S: Systemic immunization with streptococcal immunoglobulin-binding protein Sib35 induces protective immunity against group A streptococcus challenge in mice. Vaccine 2005;23:4852-4859.

-49 Norrby-Teglund A, Pauksens K, Holm SE, Norgren M: Relation between low capacity of human serum to inhibit streptococcal mitogens and serious manifestation of disease. J Infect Dis 1994;170:585-591.
50 Norrby-Teglund A, Kaul R, Low DE, McGeer A, Andersson J, Andersson U, Kotb M: Evidence for the presence of streptococcal-superantigen-neutralizing antibodies in normal polyspecific immunoglobulin G. Infect Immun 1996;64:5395-5398.

51 Agniswamy J, Lei B, Musser JM, Sun PD: Insight of host immune evasion mediated by two variants of group A streptococcus Mac protein. J Biol Chem 2004;279:52789-52796.

-52 Kawabata S, Tamura Y, Murakami J, Terao Y, Nakagawa I, Hamada S: A novel, anchorless streptococcal surface protein that binds to human immunoglobulins. Biochem Biophys Res Commun 2002;296:1329-1333.

-53 Okamoto S, Terao Y, Tamura Y, Hamada S, Kawabata S: Streptococcal immunoglobulin-binding protein Sib35 exerts stimulatory and mitogenic effects towards mouse B lymphocytes. FEMS Microbiol Lett 2008;281: 73-80.

-54 Hess JL, Porsch EA, Shertz CA, Boyle MD: Immunoglobulin cleavage by the streptococcal cysteine protease IdeS can be detected using protein $\mathrm{G}$ capture and mass spectrometry. J Microbiol Meth 2007;70:284-291.

55 Berggren K, Johansson B, Fex T, Kihlberg J, Björck L, Luthman K: Synthesis and biological evaluation of reversible inhibitors of IdeS, a bacterial cysteine protease and virulence determinant. Bioorg Med Chem 2009;17: 3463-3470

56 Berggren K, Vindebro R, Bergström C, Spoerry C, Persson H, Fex T, Kihlberg J, von Pawel-Rammingen U, Luthman K: 3-Aminopiperidine-based peptide analogues as selective noncovalent inhibitors of the bacterial cysteine protease IdeS. J Med Chem 2011 (in press).

57 Johansson BP, Shannon O, Björck L: IdeS: a bacterial proteolytic enzyme with therapeutic potential. PLoS One 2008;3:e1692.

- 58 Nandakumar KS, Johansson BP, Björck L, Holmdahl R: Blocking of experimental arthritis by cleavage of IgG antibodies in vivo. Arthritis Rheum 2007;56:3253-3260.

-59 Yang R, Otten MA, Hellmark T, Collin M, Björck L, Zhao MH, Daha MR, Segelmark M: Successful treatment of experimental glomerulonephritis with IdeS and EndoS, IgGdegrading streptococcal enzymes. Nephrol Dial Transplant 2010;8:2479-2486. 